Document downloaded from:

http://hdl.handle.net/10251/144814

This paper must be cited as:

Galindo, J.; Navarro, R.; Tarí, D.; García -Olivas, G. (05-1). Centrifugal compressor influence on condensation due to Long Route-Exhaust Gas Recirculation mixing. Applied Thermal Engineering. 144:901-909. https://doi.org/10.1016/j.applthermaleng.2018.09.005

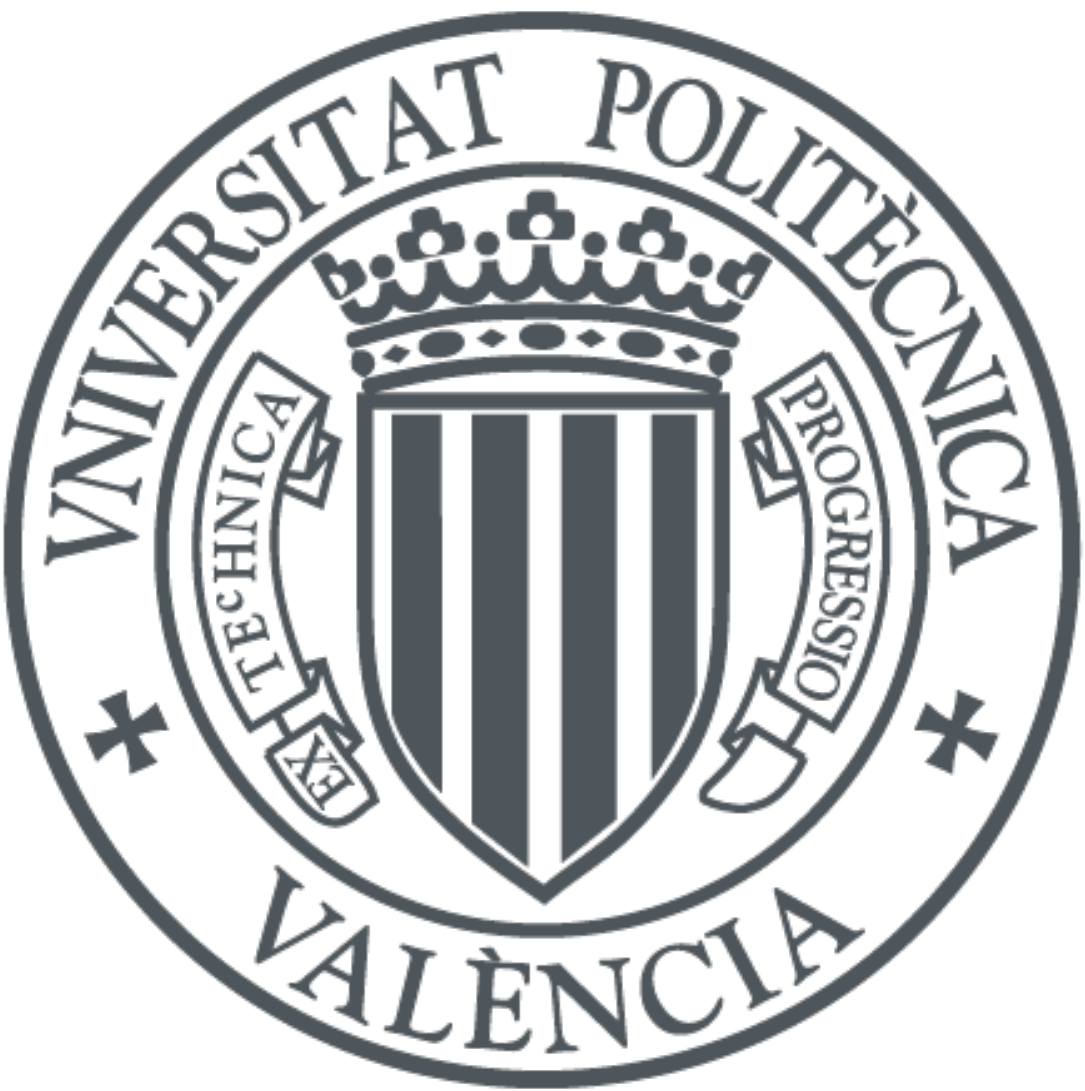

The final publication is available at

https://doi.org/10.1016/j.applthermaleng.2018.09.005

Copyright Elsevier

Additional Information 


\section{NOTICE:}

this is the author's version of a work that was accepted for publication in Applied Thermal Engineering. Changes resulting from the publishing process, such as peer review, editing, corrections, structural formatting, and other quality control mechanisms may not be reflected in this document. Changes may have been made to this work since it was submitted for publication. A definitive version was subsequently published as [1]:

\section{References}

[1] J. Galindo, R. Navarro, D. Tarí, G. García-Olivas, Centrifugal compressor influence on condensation due to Long Route-Exhaust Gas Recirculation mixing. Applied Thermal Engineering 144 (2018) 901 - 909. https: // doi .org/10.1016/j . applthermaleng.2018.09.005. 


\title{
Centrifugal compressor influence on condensation due to Long Route-Exhaust Gas Recirculation mixing
}

\author{
J. Galindo a , R. Navarro ${ }^{\text {a }}$, D. Taría,*, G. García-Olivas ${ }^{a}$ \\ ${ }^{a}$ CMT - Motores Térmicos, Universitat Politècnica de València \\ Camino de Vera, 46022 Valencia
}

\begin{abstract}
State of the art techniques for reducing pollutant emission in internal combustion engines often require local flow assessment, specially in the air management field. This work addresses the interaction between a turbocharger compressor and the 3-way long-route EGR joint, where exhaust gases of the combustion are mixed with fresh air. A validated methodology for compressor simulation is combined with a validated condensation model for this work. Numerical simulations of two different working points with three flap positions are conducted. The influence of these operating conditions on the flow field is evaluated. Particularly, there is a connection between the mixing of both steams and the generation of water condensates, responsible for the erosion of the impeller and the loss of compressor efficiency. Moreover, neglecting the impact of the compressor presence on the condensation production is shown to be of low magnitude, so that simulations without the compressor are regarded as accurate, thus reducing the computational effort by two orders of magnitude.
\end{abstract}

Keywords: Turbocharger, Computational Fluid Dynamics, Long Route EGR, Condensation

\section{Introduction}

With more and more evidences of the pollutant emissions being partially responsible for the climatic change and health issues, the regulations have

\footnotetext{
${ }^{*}$ Corresponding author. Tel.: +34 963877650

Email addresses: galindo@mot.upv.es (J. Galindo), ronagar1@mot.upv.es (R. Navarro), datade@mot.upv.es (D. Tarí), guigarol@mot.upv.es (G. García-Olivas) 
been progressively tightened during the latests years, particularly for Internal Combustion Engines (ICE). One of the most developed emission control technique used in ICE to achieve the required emission reduction is the Exhaust Gas Recirculation (EGR) [1, 2, 3]. This process consists in reintroducing back in the cylinders a fraction of the exhaust gases resulted from the combustion process mixed with fresh air. Due to this mixing, the maximum temperature is lowered during the combustion, drastically reducing the formation of nitrogen oxides [1].

Moreover, due to the common usage of turbochargers in ICE, two recirculation paths are possible. The most common configuration is called Short Route-EGR (SR-EGR), in which the intake and exhaust manifolds are directly connected, i.e., the gases are drained upstream the turbine and reintroduced downstream the compressor. Alternatively, the Long Route-EGR (LR-EGR) configuration consists in extracting the exhaust gases downstream the turbine and after-treatment elements and reintroducing them right upstream the compressor. In both cases, a cooler to reduce the temperature of the gases and valves to control the mass flow rate are usually mounted in the EGR line.

On the one hand, using the SR-EGR option reduces the pumping losses [1] and increases the turbocharger durability, since only dry air is passing through the compressor. However, the operation of the compressor is moved towards the surge limit and the distribution of exhaust gases through the different cylinders is not homogeneous [4, 5]. On the other hand, the LREGR alternative takes the gases downstream of the after-treatment elements, avoiding issues caused by the accumulation of pollutant and acidic deposition in the EGR line, the intake manifold or the inlet ports. The main problem of the LR-EGR technique is the condensation of the water vapor of the exhaust gas when its temperature is lowered below the dew point. This may occur in the EGR cooler or in the T-joint upstream the compressor, when the exhaust gases are reintroduced to the intake duct and are mixed with fresh (and potentially cold) air. Condensation in the cooler is usually found during the warming up whereas condensation in the T-joint is strongly noticeable with ambient temperatures below $10^{\circ} \mathrm{C}$ [6]. Appearance of liquid water upstream the compressor is critical if droplets reach the impeller. The impact velocity of the droplets against the impeller is high enough to produce erosion on the leading edges, reducing the compressor efficiency and posing a risk to the turbocharger integrity if produced continuously, as seen by Karstadt et al. 7]. 
Due to the relevance of this issue, it is interesting to analyze the condensation phenomenon in order to find potential solutions. Condensation depends on the flow distribution and turbulence, i.e., how the streams mix. So, a thorough analysis should be addressed using a 3D-CFD approach. Previously, different authors have dealt with similar stream mixing problems [8, 9, 10], condensation modeling [11, 12] or both phenomena [13]. 3D-CFD has shown to be also a good tool for researchers to analyze the flow field of centrifugal compressors [14, 15]. In the particular case of condensation produced in a LR-EGR T-joint, the compressor presence as an active element on the process may be of importance. Unfortunately, modeling the whole compressor along with the LR-EGR T-joint with accuracy implies a computational effort remarkably larger than simulating only the T-joint, as will be shown in Section 4.4.

This work is devoted to assess the influence of the operating conditions and the the intake counter-pressure flap angle of a LR-EGR T-joint in the mixing process and generation of condensates, which is responsible for worsening the compressor efficiency by producing erosion on the leading edges of the compressor. Due to the difficulty of performing local measurements without being too intrusive, CFD simulations are carried out using the commercial code STAR-CCM+ [16]. The numerical setup is obtained from an experimentally validated previous work and the condensation model used was also validated with experimental data. The impact on computational effort of considering the compressor will be addressed as well.

In Section 2 the mesh and simulation setups will be detailed and the condensation model summarized. Then, the methodology, including the operating points and specific geometrical configurations, will be explained in Section 3. The results and the discussion are written in Section 4 and finally, the concluding remarks are highlighted in Section 5.

\section{Numerical configuration}

\subsection{Geometries and mesh}

As aforementioned, there are two main regions of interest in the current study; the LR-EGR junction and the compressor, which together form the complete geometry, as shown in Fig. 1. The particular LR-EGR junction selected for this work is a T-joint, as can be seen in Fig. 2. It has two perpendicular inlets, one for the intake of fresh cold air (left branch) and one for the warm and humid EGR (bottom branch), with a counter-pressure 
flap on each duct for controlling the ratio of mass flow rates (known as EGR rate). The length of the intake pipes, from each flap to their inlet boundary is three corresponding diameters [17]. The T-joint discharges into the compressor wheel (right branch of Fig. 2). Figure 3 shows that the complete geometry of the compressor is considered, i.e., $360^{\circ}$-resolved wheel, diffuser, volute and outlet duct, which is 5 diameters long [18]. There will be two sets of simulations: complete domain (T-joint with compressor; Fig. 1) and reduced domain (T-joint alone; Fig. 2). For the latter, the section at the end of the cone is extruded one and a half diameters, thus reducing the impact of the boundary condition on the solution. The angle of the EGR flap is fixed and the set of angles used for the intake flap will be discussed in Section 3 .

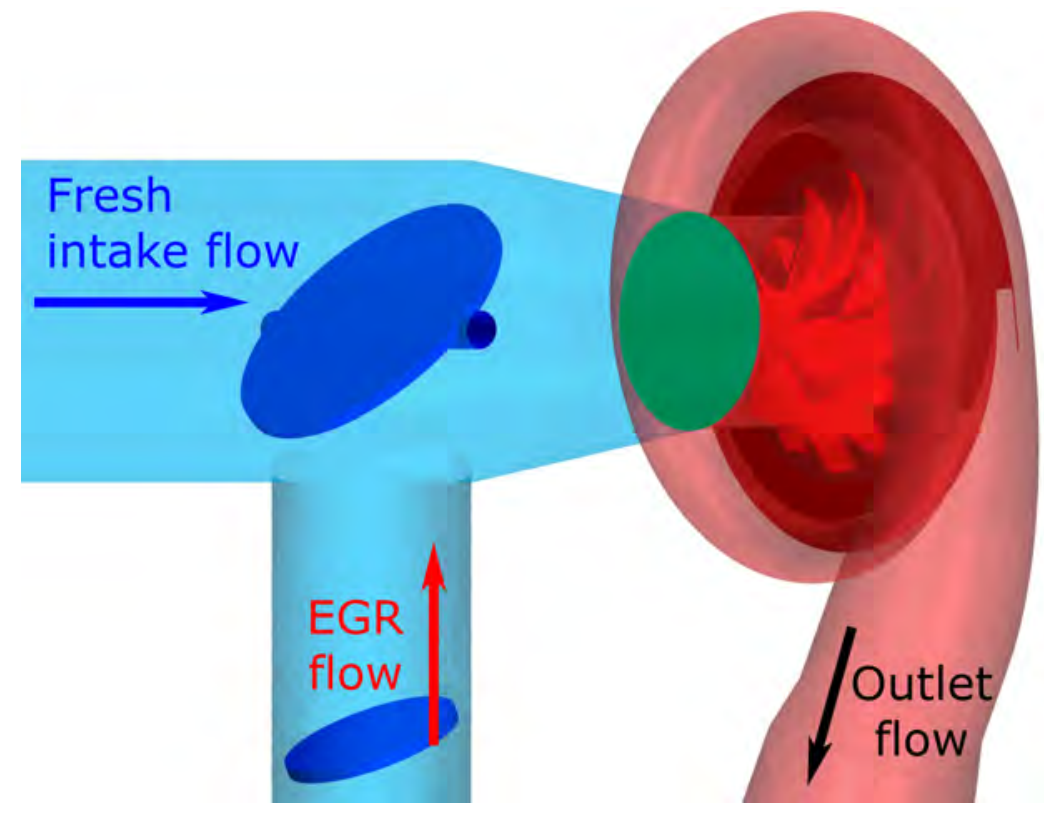

Figure 1: Geometry included in the complete domain.

For the sake of consistency, the meshing approach is kept the same for both regions. The meshing methodology corresponds to the validated approach proposed by Broatch et al. [19]. The meshes are composed of polyhedral cells, with a base size between $0.8 \mathrm{~mm}$ for the critical regions such the compressor wheel and $3 \mathrm{~mm}$ for the inlet and outlet ducts. Prism layers are generated on the walls, which improve the solution of the boundary layer. The $y^{+}$value on the walls is about 1 , assuring a proper solving of 


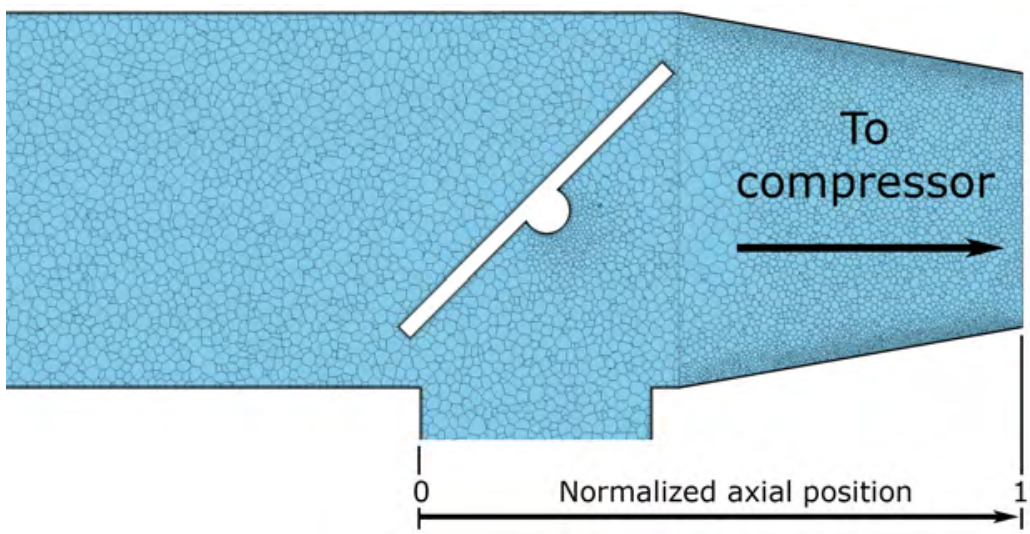

Figure 2: LR-EGR T-joint with cross-section of mesh and normalized axis for postprocessing.

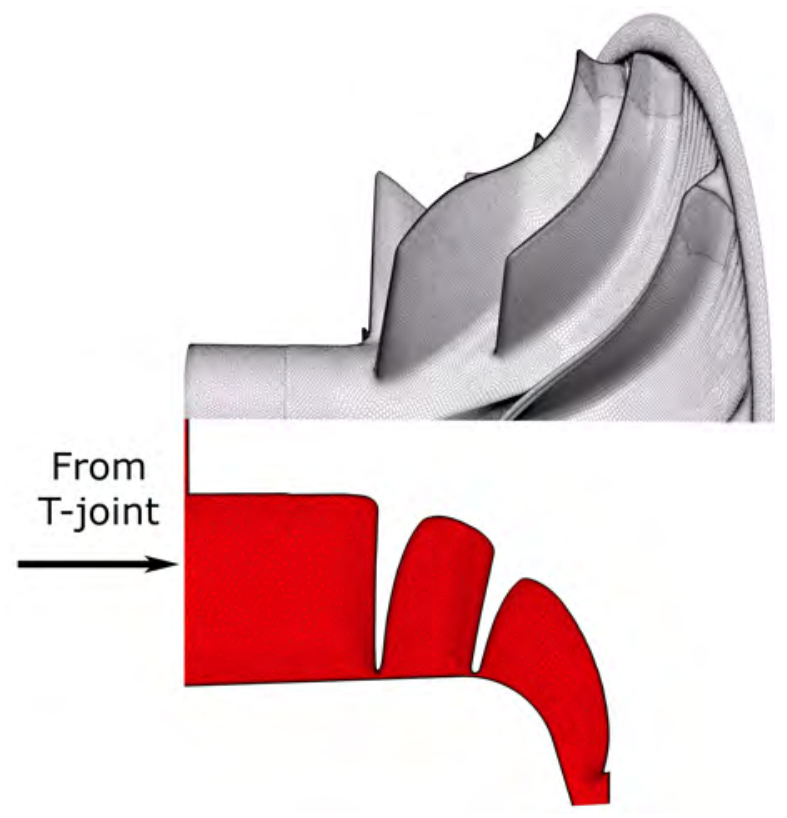

Figure 3: Compressor domain and impeller mesh.

the near-wall flow [20]. Global meshing characteristics are in agreement with those corresponding to the compressor mesh independence study performed by Navarro [18], since they are more restrictive than the meshing setup from the LR-EGR work. Navarro tested three different meshes for a similar tur- 
bocharger compressor, particularly with 4, 9 and 18 million cells. The results showed similar global variables among all three cases, but a poorly resolved aero-acoustic signature for the coarser mesh, so it was concluded that the 9 million cell mesh was enough for shedding accurate results. Applying this configuration to the current work, the meshes of the complete cases have $12 \mathrm{M}$ cells and the reduced cases (T-joint only) have $0.75 \mathrm{M}$ cells.

\subsection{Setup and boundary conditions}

The CFD methodology developed by Broatch et al. [19] is employed for setting the numerical configuration of the simulations conducted in this work. The CFD model [19] was validated with experimental measurements on a continuous flow turbocharger test rig, showing a good agreement not only in compressor global performance indicators but also in acoustic measurements.

The flow is set as multicomponent ideal gas, considering dry air and water vapor as components. The segregated solver of STAR-CCM+ [16] is chosen. For the turbulence approach, the Detached Eddy Simulation (DES) model with the $k-\omega$ SST submodel is used [21]. Broatch et al. [19] also considered URANS, concluding that DES predicted the compressor behavior with higher fidelity. Second-order time discretization is considered, rotating the compressor mesh 4 degrees per time step, which results in a time-step size of $4.2 \cdot 10^{-6} \mathrm{~s}$. Navarro [18] proved this time-step size was enough for an accurate prediction of the global variables. Walls are considered adiabatic taking into account that the low conductivity of the EGR junction material and high velocities in the compressor lead to a negligible impact of the heat transfer with the surroundings.

Mass flow inlet boundaries are selected in both fresh air and EGR inlets, specifying additionally the total temperature and the mass fraction of each component. Concerning the turbulent specification, an intensity of 0.04 and a length scale of $3.5 \mathrm{~mm}$ are set. For the cases with the compressor, the values of compressor speed and outlet pressure are required. A radial equilibrium profile is employed at the outlet boundary [22. The ratio between the inlet mass flow rates fixes the EGR rate, and the mass fraction of the gas components sets their specific humidity. The values of the boundary conditions are obtained from experimental tests performed by Tarí [17].

For the reduced-domain simulations, the outlet pressure values were not actually measured. Instead, the cases with the complete domain are run first and the pressure at a plane at the end of the inlet cone is averaged and used as a constant and homogeneous value for the reduced cases. This pressure 
depends on the operating point and it is not known beforehand, but Tarí [17] showed that sensitivity of the solution to the T-joint outlet pressure is low (1\% change in condensation rate for a deviation of $1000 \mathrm{~Pa}$ in the boundary condition).

\subsection{Condensation submodel}

Condensation modeling is a key feature for the current analysis. The model selected was developed and verified by Serrano et al. [6] and then validated by Tarí [17] through an experimental campaign of durability tests. For a proper understanding of the phenomena that takes place in the LREGR joint, a brief description of this physic process and the approach of the condensation model is provided.

Due to the non-linear capacity of the dry air to accept water vapor with the temperature, it is possible that, when two undersaturated streams with different psychrometric conditions (temperature, humidity and pressure) are mixed, the resulting gas becomes oversaturated, and thus condensation is produced. This particular condensation mechanism is potentially given in the bulk flow of a LR-EGR T-joint, thus forming small droplets that are dragged by the flow and grow as coalescence and further condensation occurs.

The employed model disregards the behavior of the actual droplets, since a Lagrangian approach or a sophisticated ad hoc model would be then needed, substantially increasing the computational effort required. Instead, liquid water is tracked using a passive scalar which does not affect the continuum flow once it is generated. Due to the reduced volume fraction of the liquid component, this assumption does not have an impact on the results. Further descriptions of the assumptions and simplifications made in model are performed in the works of Serrano et al. [6] and Tarí [17].

Condensation is modeled by including custom source terms in STAR-CCM+ [16]. The relative humidity of the domain is calculated first. Condensation is triggered when oversaturated conditions are detected in a given cell. A weighing equation is used considering the enthalpy and humidity of the initial conditions to calculate the amount of vapor content that should be subtracted from the cell to achieve saturated conditions. The source term for the vapor mass component is described in Equation 1.

$$
S_{v a p}=\frac{\rho Y_{a i r}\left(w_{f}-w_{1}\right)}{\Delta t}
$$


The specific humidity subtraction denotes the difference between the initial and the final humidities. The characteristic time of the denominator is related with the residence time of a particle in a given cell.

Known the mass source term, the energy source term is calculated considering the latent heat of vaporization released during the condensation, originating an isenthalpic process. In Equation 2 is showed this relation.

$$
S_{\text {ener }}=S_{\text {vap }}\left(L-c_{p_{\text {vap }}} T\right)
$$

The sensible heat of the condensed vapor mass needs also to be decreased to assure the energy equilibrium of the remaining gas.

Additionally, the momentum of the decreased vapor mass is considered. for this, the velocity vector is multiplied by the vapor source term, as seen in Equation 3 .

$$
S_{\text {mom }}=S_{\text {vap }} \vec{v}
$$

Finally, the passive scalar is generated inversely to the decrease of the condensed vapor mass component, virtually tracking the presence of the liquid water as it was behaving as a gas component, with convection and diffusion. Tarí [17] validated this model performing experimental durability tests. In a steady rig, hot humid air was used as a surrogate of EGR gases and a cold chamber was employed to generate very cold ambient conditions, from where the compressor sucked a given mass flow rate. Using a similar T-joint to the one employed in this work, the hot stream was mixed with the cold air upstream the compressor. Tarí [17] conducted 50-hour tests to assess the impact of the condensates generated on the compressor wheel wear, finally correlating the condensation rate predicted by the CFD model with this impeller erosion. A direct agreement was found between the erosion observed and the predicted condensation rate, thus validating the model used in this work.

\section{Methodology}

\subsection{Numerical campaign}

A combination of different operating conditions and geometries are analyzed. Two operating points are combined with three flap angle positions. The first operating point has high intake mass flow rate, low EGR rate and high compressor speed, the second has a low intake mass flow rate, high EGR 
rate and low compressor speed. The numeration of the cases and the flap angles are shown in the Table 1. To assess the influence of the compressor on the condensation process, all six previous configurations are run considering the domains introduced in section 2.1, i.e., complete geometry (with compressor) and reduced geometry (without compressor). In these 12 simulations several performance indicators are employed to conduct quantitative comparisons. They are described in Section 3.2 .

Table 1: Simulation campaign.

\begin{tabular}{cccccc}
\hline Op. point & $\mathbf{T}_{\text {in }}$ & $\mathbf{T}_{\text {EGR }}$ & $\dot{\mathbf{m}}_{\mathbf{a}}$ & EGR rate & Comp. speed \\
\cline { 1 - 4 } $\mathrm{A}$ & $-10{ }^{\circ} \mathrm{C}$ & $90{ }^{\circ} \mathrm{C}$ & $\uparrow$ & $15 \%$ & \\
$\mathrm{~B}$ & $-10{ }^{\circ} \mathrm{C}$ & $50{ }^{\circ} \mathrm{C}$ & $\downarrow$ & $35 \%$ & \\
\hline Case & Intake flap angle $\left(^{\circ}\right)$ & Op. point $(-)$ \\
\hline 45A & 45 & $\mathrm{~A}$ \\
15A & 15 & $\mathrm{~A}$ \\
0A & 0 & $\mathrm{~A}$ \\
45B & & 45 & $\mathrm{~B}$ \\
15B & & 15 & $\mathrm{~B}$ \\
0B & & & $\mathrm{B}$ \\
\hline
\end{tabular}

\subsection{Parameters analyzed}

The consideration of the passive scalar for tracking the generated condensates allows the calculation of the liquid water mass flow rate $\left(\dot{m}^{l i q}\right)$ that passes through a given section with little additional effort. Thus, by performing this operation along different sections between the EGR discharge duct and the compressor inducer, it is possible to analyze and quantify the influence of the compressor on the mixing process and determine the impact of neglecting the compressor from the simulation. Additionally, an overview of the influence of using different operating conditions and flap angles will be possible through this analysis. Differences in condensation rate between the complete and the reduced cases will be displayed in absolute and relative values, which are calculated following Equations 4 and 5 respectively.

$$
\epsilon_{a b s}=\dot{m}_{c o m p}^{l i q}-\dot{m}_{r e d}^{l i q} \quad\left(\frac{g}{h}\right)
$$




$$
\epsilon_{r e l}=\frac{\dot{m}_{c o m p}^{l i q}-\dot{m}_{r e d}^{l i q}}{\dot{m}_{c o m p}^{l i q}} \cdot 100
$$

Condensation depends on how the streams are mixed, which in turn is determined by the flow structures. The mechanisms that set the main flow structures will be then studied and discussed in Section 4 .

Analogously, each flow pattern sets a different pressure distribution. Pressure scalar field on the section at the end of the cone (boundary in the right side of Fig. 21 will be analyzed to provide useful information regarding the predominant mechanism that controls the flow structure at the inducer.

\section{Results}

\subsection{Axial evolution of condensation rate}

The mass flow rate of condensed water (condensation rate) from the EGR discharge duct to the end of the inlet cone for all the cases are shown in Figures 4 (op. point A) and 5 (op. point B). The vertical axis shows the condensation rate normalized with dry air mass flow rate and the horizontal axis displays the axial position normalized with the distance between the EGR discharge duct and the end of the inlet cone (see Fig. 2). This is the critical region where the condensation is produced before reaching the compressor wheel. After entering the inducer, droplets may impact other components, deposit over the surfaces and even re-evaporate as the gas is compressed and heated up. However all this latter phenomena are not considered in the model since the most harmful mechanism is the impact of the droplets against the leading edges of the compressor.

\subsection{Analysis of complete cases}

In first place, the results of the complete case are analyzed. Solid lines of Fig. 4 show the results of the cases with of operating point A. It is noticeable that in this case, where the EGR rate is low, small intake flap angles produce the same results of condensation, being the differences between $0^{\circ}$ and $15^{\circ}$ almost non-existent. Conversely, a high flap angle enhances condensation generation. Figure 5 shows the results of operating point B. In this case, the high EGR rate that characterizes these cases clearly controls the mixing and the condensation, producing similar results for all three cases. Nevertheless, the highest flap angle of $45^{\circ}$ still produces at the end of the inlet cone the greatest condensation rate. 


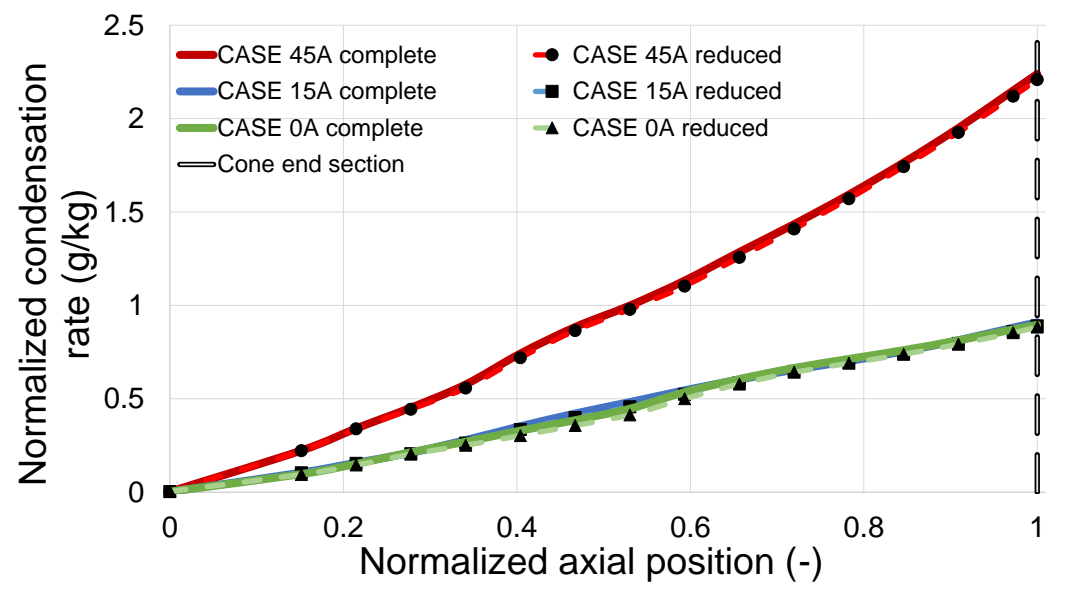

Figure 4: Condensation rate of operating point A cases.

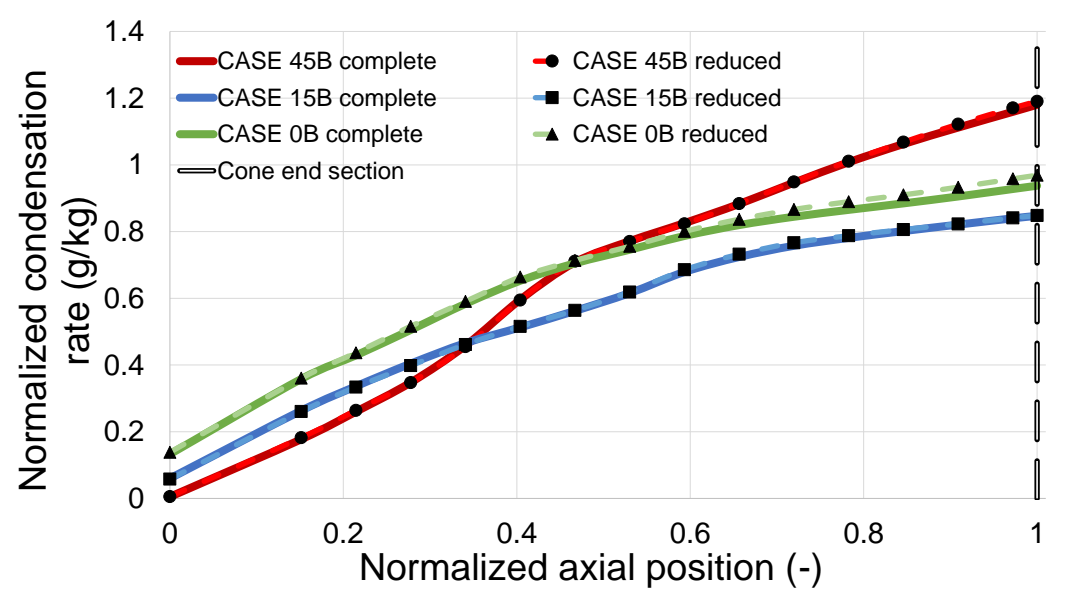

Figure 5: Condensation rate of operating point B cases.

The influence of the EGR rate and flap angle on the mixing process is illustrated in Figs. 66 and 7, where a Line Integral Convolution (LIC) [23] representation of the time-averaged temperature scalar over a longitudinal section, together with time-averaged velocity vectors, are displayed. Figure 6 is devoted to the cases $15 \mathrm{~A}$ and $45 \mathrm{~A}$, with lower EGR rate than their counterparts 15B and 45B, depicted in Fig. 7. In these figures it can be observed the EGR stream entering the T-joint from the bottom branch with higher temperature. As both streams mix, the temperature gradient at a given cross-section is reduced, which, considering the condensation rate results in 

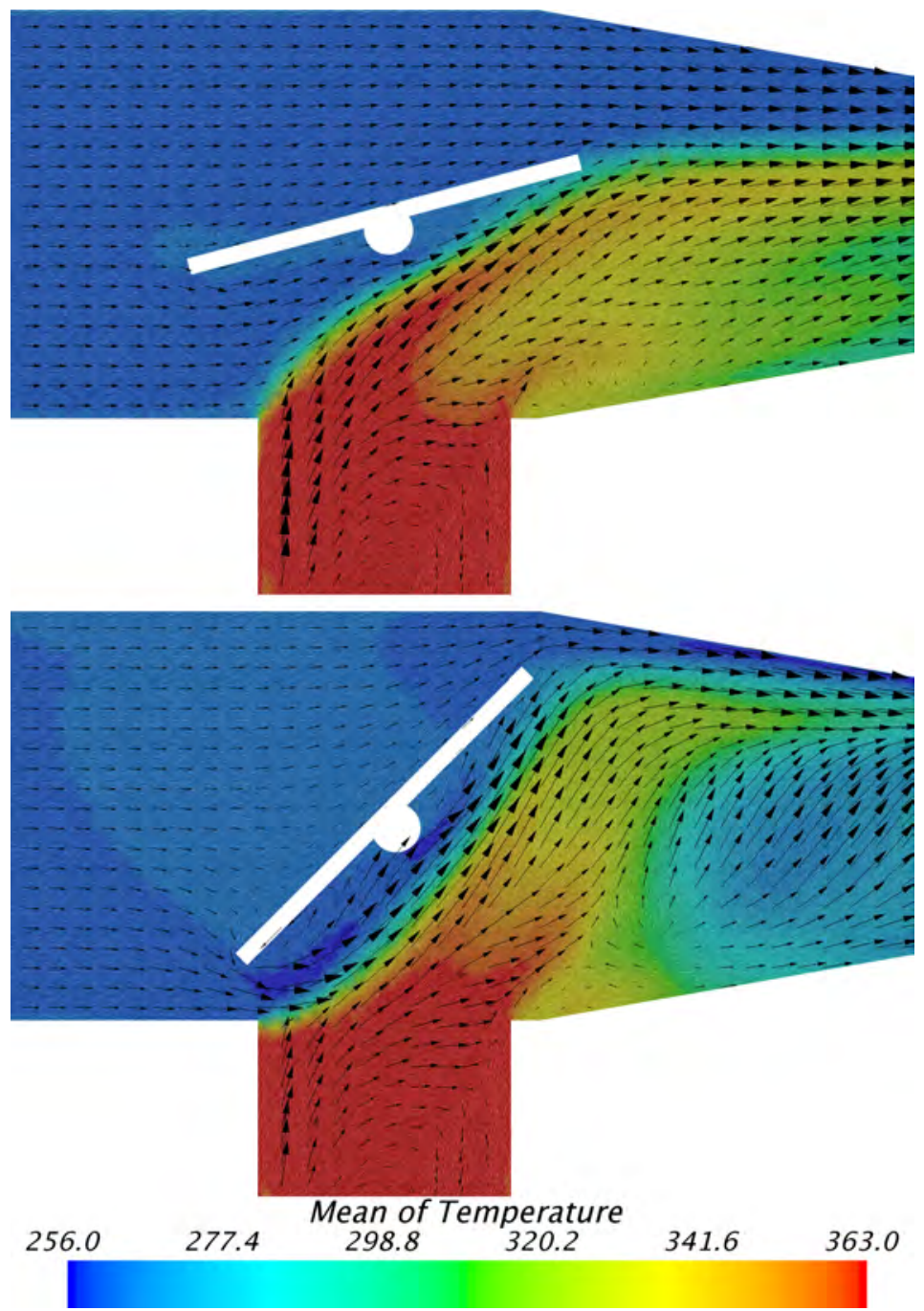

Figure 6: Line integral convolution of temperature and velocity vectors at longitudinal section for cases 15A (top) and 45A (bottom).

Figs. 4 and 5 , prove that the mixing of the streams and the condensation produced is correlated.

For the low EGR rate cases on Fig. 6, the high angle of the flap (bottom of the picture) induces high vorticity and turbulent structures which enhance condensation generation. Moreover, the low momentum region created by the valve reduces pressure, so that the EGR flow is directed upwards, further contributing to mixing of streams and condensation. Also, strong 

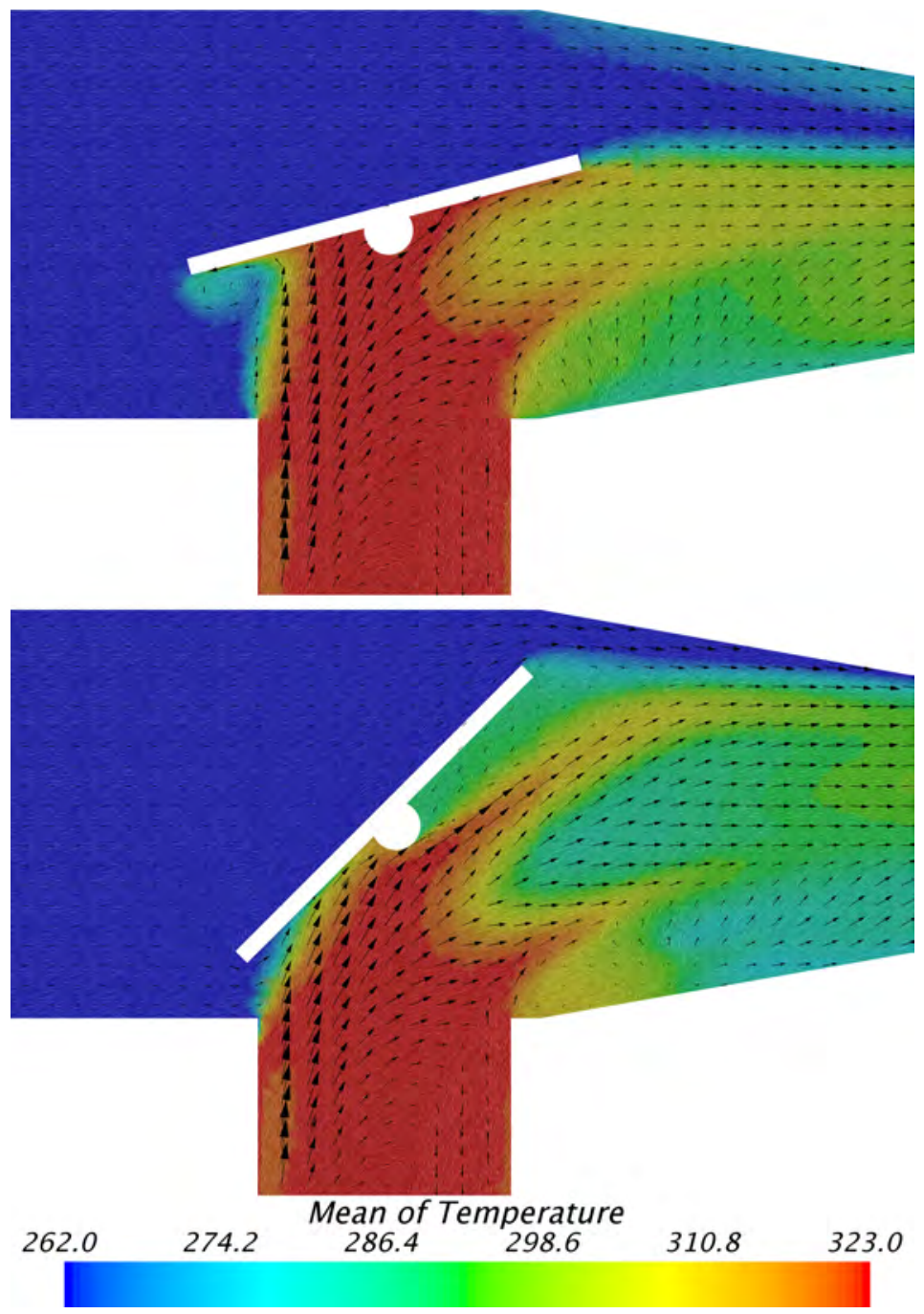

Figure 7: Line integral convolution of temperature and velocity vectors at longitudinal section for cases 15B (top) and 45B (bottom).

counter-rotating swirling structures are created by the flap and convected downstream, which extend the mixing region. On the contrary, when the flap angle is reduced (upper side of Fig. 6), the intake flow impinges and smoothly deflects the EGR stream towards the compressor, avoiding excessive mixing. For operating point A, Figure 4 suggests that there should be a threshold of low-end flap angle that does not affect the flow structure enough to have repercussions on the mixing. 
High EGR rate cases corresponding to working point B, depicted in Fig. 7. show a lower influence of flap angle because the penetration of the EGR stream is higher, similarly impacting against the flap regardless of its angle. Part of the EGR stream may even surround the flap and overcome to the upper side towards the inlet face, advancing the condensation production, as can be seen in case 15B (top of Fig. 7). The condensation rate evolution of case $0 \mathrm{~B}$, depicted in the Fig. 5, shows an offset of condensation rate at the origin of the axial position, implying that condensates are being produced upstream the EGR duct, due to the aforementioned issue. This issue exists for operating conditions combining lower flap angles and high EGR rates.

\subsection{Comparison of reduced and complete cases}

The impact of the compressor on the mixing process and condensation generation can be assessed by comparing the results of condensation rate of the complete cases with their analogous with reduced geometries. Figures 4 and 5 show such results, in which dashed lines with symbols represent the reduced counterparts of the complete cases. Generally, the axial profiles of condensation rate predicted without compressor follow the ones corresponding to the complete cases with great agreement. Only a slight discrepancy is found in case $0 \mathrm{~B}$, but the reduced case prediction is still quite close to the solution of the complete geometry. The absolute and relative errors of condensation rate, calculated using Equations 4 and 5, are evaluated at the end of the inlet cone (normalized axial position $=1$ in Figures 4 and 5 and showed in Table 2 .

Table 2: Condensation rate error in the cross-section at the end of the inlet cone.

\begin{tabular}{c|c|c}
\hline Case & $\epsilon_{\text {abs }}(\mathrm{g} / \mathrm{h})$ & $\epsilon_{\text {rel }}(\%)$ \\
\hline 45A & -8.58 & -1.37 \\
15A & -5.37 & -2.11 \\
0A & -3.44 & -1.37 \\
45B & 1.31 & 0.86 \\
15B & 0.15 & 0.14 \\
0B & 4.05 & 3.34 \\
\hline
\end{tabular}

The relative errors found are all below 4\%. Thus, the compressor does not significantly affect the generation of condensates. In spite of not playing 
an important role on the condensation production, it is worth analyzing why its influence is so small.

As can be seen in Figure 8, when comparing the water distribution at the end of the mixing region for the complete (top) and reduced (bottom) cases, it is noticed that such strong patterns are unlikely to be perturbed by the compressor influence. In operating point $\mathrm{A}$, where the intake mass flow is relatively high, strong and stable vortices are formed downstream of the flap, fixing this pattern from that point and explaining why both flow structures and condensation rate are almost identical.

On the contrary, Figure 9 shows the comparison of flow structure between the complete (top) and reduced (bottom) 45B cases. Operating point B has a lower intake mass flow rate that is combined with a higher EGR flow, what causes a weak and unsteady pattern that is likely permeable to external interactions such as the influence of the compressor. Note that velocity vectors in Fig. 9 are much smaller than those displayed in Fig. 8, and so are secondary flows (velocity vectors in Figs. 8 11 use the same scaling factor). That is why the condensation patterns in Fig. 9 are not the same for the complete and reduced case. However, due to the actual operating conditions of the compressor (low speed and compression ratio), its perturbation upstream the wheel is also weak, so the impact on secondary flows (and thus condensation) is only slight. There could be operating conditions where the impact could be greater, however these conditions are not in the common working range of the coupling between the turbocharger and the EGR demand [24].

An additional approach to detect predominant flow patterns resides in observing the pressure contours. Cases with strong flow structures generated during the mixing process usually have defined vortices with elevated pressure gradients. The pressure range of a given section plane is therefore defined as: $p_{\text {range }}=p_{\max }-p_{\min }$, where all pressures are time-averaged. In Table 3 the pressure range at the section at the end of the inlet cone are provided, for both complete and reduced cases.

There are two phenomena mainly responsible for creating in-plane pressure gradients that would drive secondary flows. First, regarding the valve, higher flap angles and higher mass flow rates (and velocities) imply a higher pressure gradient. This occurs in case 45A, where the high mass flow and the high flap angle produce two strong counter-rotating vortices with low pressure in their centers, masking the rest of the pressure perturbations, as can be seen in the comparison of Figure 10. Second, concerning the impeller, the closeness of the nut of the wheel to this section drives the gas stream 

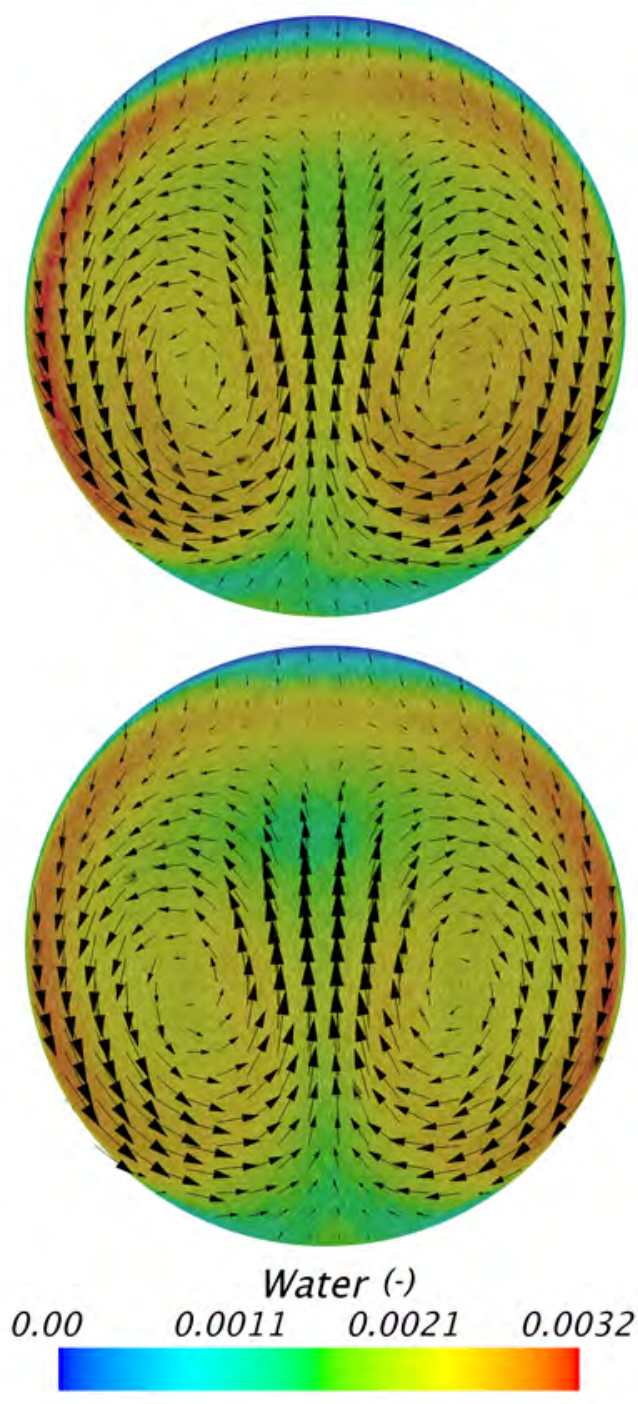

Figure 8: Cross-section time-averaged water fraction contours and in-plane velocity vectors comparison at the end of the inlet cone of case 45A. Complete case on top and reduced geometry on bottom.

away from the center of the duct, lowering the velocity and increasing the pressure near the central part. This phenomenon increases with the mass flow rate and causes a higher pressure gradient compared with a simulation without the compressor and the nut.

In the rest of the cases, where the flow structures at the mixing region are 

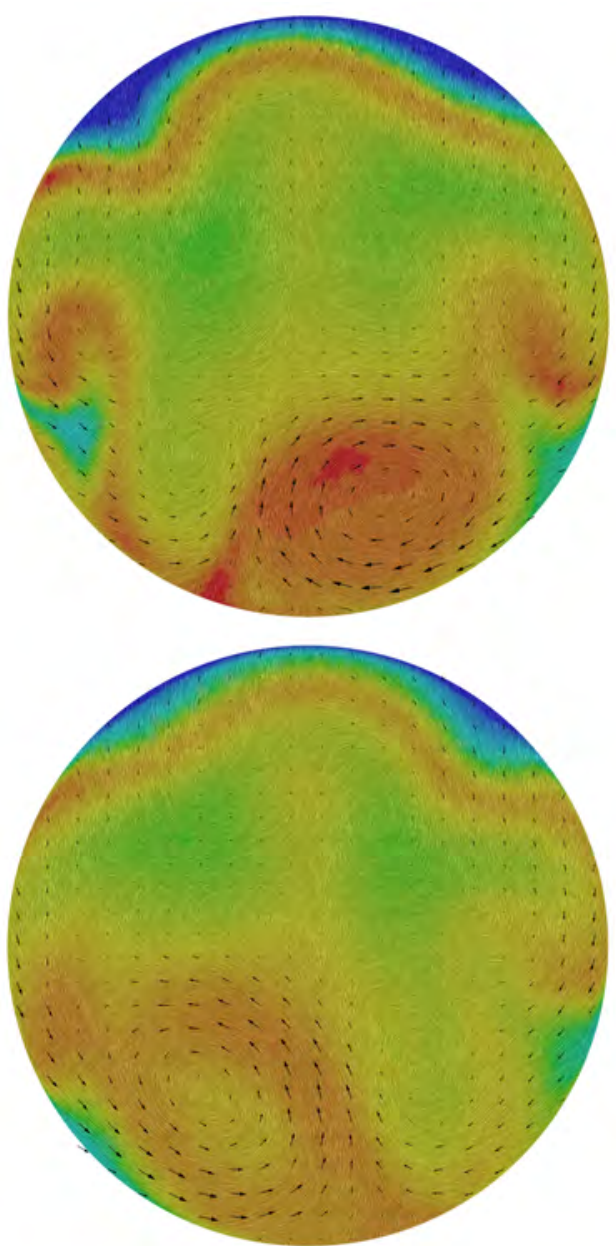

Water (-)

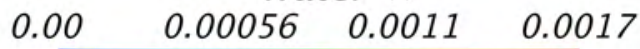

Figure 9: Cross-section time-averaged water fraction contours and in-plane velocity vectors comparison at the end of the inlet cone of case 45B. Complete case on top and reduced geometry on bottom.

not as dominating as in case $45 \mathrm{~A}$, the influence of the compressor is clearer. A lower pressure range can be observed in Fig. 11, where the flap angle is set at $0^{\circ}$. The presence of the nut in the central part of the complete simulation causes the pressure to be noticeable higher, while in the reduced case the pressure distribution is homogeneous. Even though the pressure 
Table 3: $p_{\text {range }}$ at the end of the inlet cone section.

\begin{tabular}{c|c|c}
\hline Case & Complete Case $\mathbf{( P a )}$ & Simplified Case $\mathbf{( P a )}$ \\
\hline 45A & 5360 & 5710 \\
15A & 1812 & 1239 \\
0A & 2031 & 1221 \\
45B & 618 & 444 \\
15B & 351 & 279 \\
0B & 486 & 262 \\
\hline
\end{tabular}

distribution at the complete and reduced cases is not identical, the impact of this difference on the secondary flows is limited. Figure 11 shows that in both cases the velocity vectors are of the same magnitude, much smaller than the ones depicted in Fig. 10 indicating weaker secondary flows.

\subsection{Computational effort}

To assess the impact on computational effort of reducing the domain, the actual time each simulation took to calculate a single time step is summed up in Table 4, considering both complete and reduced cases and using ten cores for all the cases. For the complete cases, the time needed to turn the rotor region mesh after each time step is included as well.

Table 4: Comparison of computational effort.

\begin{tabular}{l|cc}
\hline Geometry & Reduced & Complete \\
\hline Elapsed time/time step (s) & 21 & 447 \\
Number of time steps (\#) & 1000 & 3000 \\
Turnaround time (days) & 0.24 & 15.5 \\
\hline
\end{tabular}

A time step of a complete simulation takes about 20 times more than a time step of a simulation with only the T-joint geometry. Furthermore, for properly converging a compressor simulation, several wheel rotations must be carried out and due to the chosen time step, the final time each complete simulation needs to be converged is around two weeks, with a 10-core parallel computation. Conversely, a reduced case only takes six hours to be completely solved, even less if the solution can be solved using a steady state time discretization, as seen by Tarí [17]. 


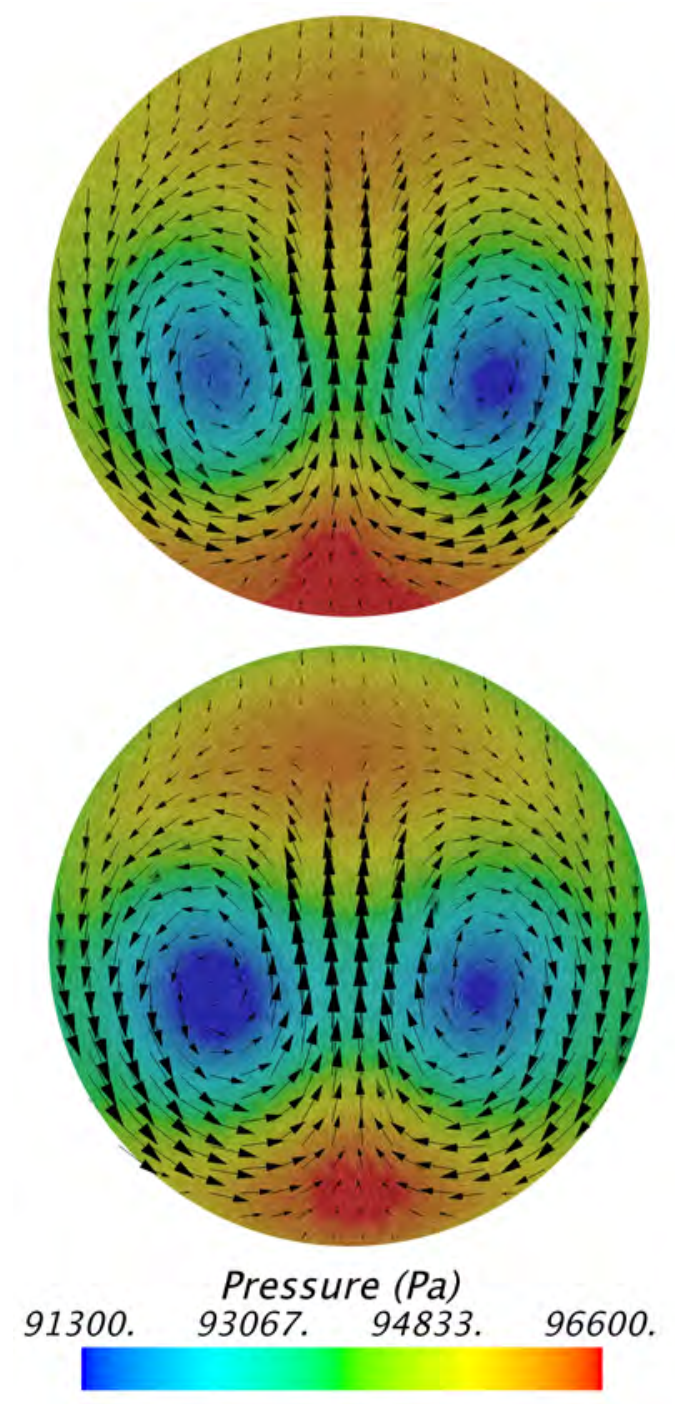

Figure 10: Cross-section scalar of pressure comparison at the end of the inlet cone of case $45 \mathrm{~A}$. Complete case on top and reduced geometry on bottom.

\section{Concluding remarks}

After discussing the results and assessing the impact of decoupling the compressor from the simulation in terms of computational effort, the following conclusions are obtained.

- Condensation distribution at the compressor similarly depends on both 


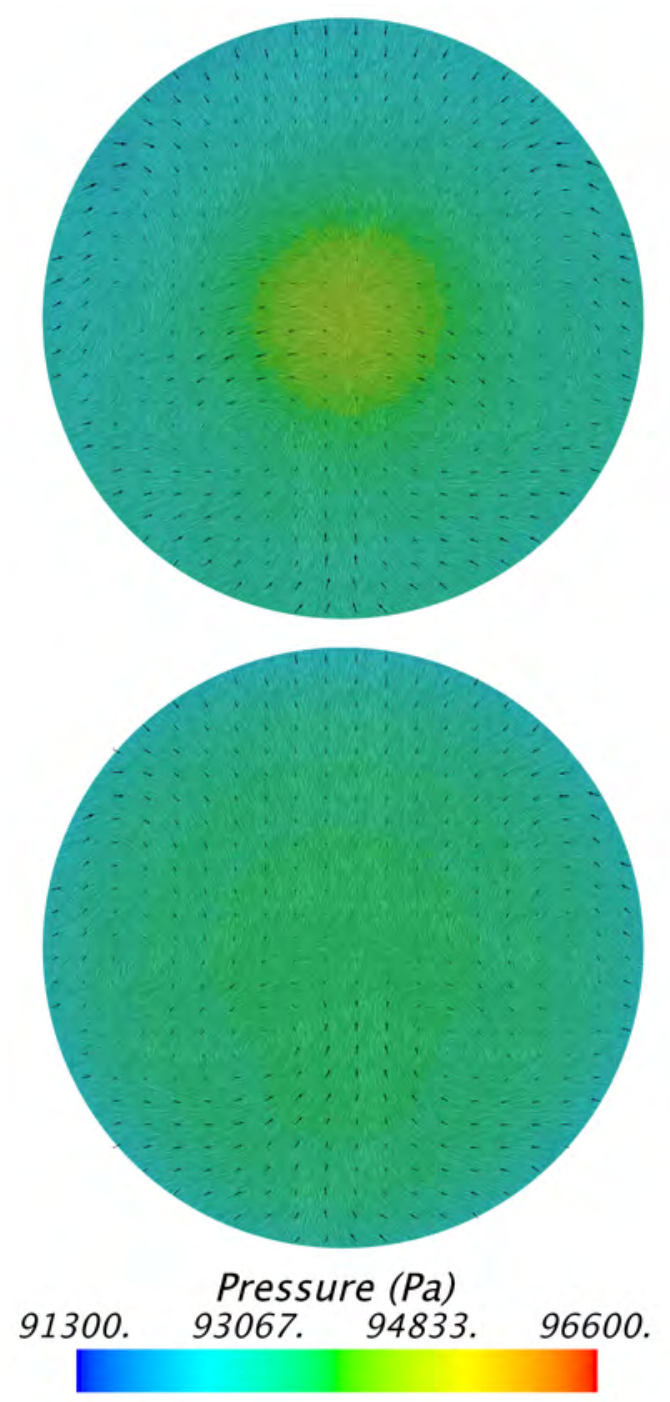

Figure 11: Cross-section scalar of pressure comparison at the end of the inlet cone of case 0A. Complete case on top and reduced geometry on bottom.

the geometry of the LR-EGR T-joint and on the operating conditions. When the intake flap angle is high, very turbulent flow structures are formed and the condensation production is increased.

- The comparison of the results shows that the compressor has minimal influence on the condensation prediction. Only when the flow structure 
is not being altered by the flap or the EGR stream and backflows appear at the inducer, some relative error may be noticeable, and even in these cases the error is below $4 \%$ for the studied cases.

- By neglecting the influence of the compressor in the T-joint domain, the computational effort required is reduced by two orders of magnitude, allowing an extensive analysis of the condensation focused only on this part.

- The aforementioned conclusion concerning the possibility of decoupling the LR-EGR from the compressor can also be applied to the experimental field. If the compressor is not placed a the outlet of the T-joint in a test bench, the access to different measurement techniques become possible, such as measurements of local flow velocity, temperature or even humidity. Laser techniques, such as PIV [25, 26, 27] or LDV [28, 29], widely used by different researchers, may also be used in this case, providing information enough to fully characterize the flow pattern and the condensation evolution.

Future works on this topic are addressed to develop a simple model that predicts the value of the outlet pressure of the T-joint for the aforementioned simulations. A proper model should consider air and EGR mass flow rates, upstream pressures, flap angles and other parameters. The model should be then validated with additional simulations and experimental tests.

\section{Acknowledgements}

Daniel Tarí is partially supported through contract FPI-S2-2015-1095 of Programa de Apoyo para la Investigación y Desarrollo (PAID) of Universitat Politècnica de València.

\section{References}

[1] J. M. Desantes, J. M. Luján, B. Pla, J. A. Soler, On the combination of high-pressure and low-pressure exhaust gas recirculation loops for improved fuel economy and reduced emissions in high-speed directinjection engines, International Journal of Engine Research 14 (1) (2013) 3-11. doi:10.1177/1468087412437623. 


\section{List of Symbols}

$c_{p} \quad$ isobaric specific heat capacity $J \cdot k g^{-1} \cdot K^{-1}$

$L$ latent heat

$J \cdot k g^{-1}$

$N \quad$ compressor speed

rpm

$\dot{m}$ mass flow rate

$g \cdot h^{-1}$

$p \quad$ pressure

$\mathrm{Pa}$

$S$ source term

$T$ temperature

$\vec{v} \quad$ velocity

$w \quad$ specific humidity

$Y$ mass fraction

$\Delta t$ characteristic time

$\epsilon$ error

$\rho$ density

$K$

$m \cdot s^{-1}$

$g_{\mathrm{H}_{2} \mathrm{O}} \cdot k g_{\text {air }}^{-1}$

$s$

$\%$

$k g \cdot m^{-3}$

Sub- and Superscripts

abs absolute

air dry air

comp complete

ener. energy

mom. momentum

liq liquid water

red reduced

rel relative

vap vapor component

\section{List of abbreviations and acronyms}

3D three dimensional

CFD Computational fluid dynamics

DES Detached eddy simulation

EGR Exhaust gas recirculation

ICE Internal combustion engines

LIC Line integral convolution

LR-EGR Long-route EGR

Op operating

SR-EGR Short-route EGR 
[2] D. T. Hountalas, G. C. Mavropoulos, K. B. Binder, Effect of exhaust gas recirculation (EGR) temperature for various EGR rates on heavy duty DI diesel engine performance and emissions, Energy 33 (2) (2008) 272-283. doi:10.1016/j. energy.2007.07.002.

[3] N. Ladommatos, S. Abdelhalim, H. Zhao, The effects of exhaust gas recirculation on diesel combustion and emissions, International Journal of Engine Research 1 (1) (2000) 107-126. doi:10.1243/ 1468087001545290 .

[4] J. Luján, B. Pla, S. Moroz, G. Bourgoin, Effect of low pressure EGR on gas exchange processes and turbocharging of a HSDI engine, in: Proceedings of the conference on thermo-and fluid-dynamic processes in diesel engines (THIESEL 2008), Valencia, Spain, paper E, Vol. 2, 2008.

[5] L. Cornolti, A. Onorati, T. Cerri, G. Montenegro, F. Piscaglia, 1D simulation of a turbocharged Diesel engine with comparison of short and long EGR route solutions, Applied Energy 111 (2013) 1-15. doi: 10.1016/j.apenergy.2013.04.016.

[6] J. Serrano, P. Piqueras, R. Navarro, D. Tarí, C. Meano, Development and verification of an in-flow water condensation model for 3D-CFD simulations of humid air streams mixing, Computers \& Fluids 167 (2018) 158 - 165. doi:10.1016/j.compfluid.2018.02.032.

[7] S. Karstadt, J. Werner, S. Münz, R. Aymanns, Effect of water droplets caused by low pressure EGR on spinning compressor wheels, in: Aufladetechnische Konferenz, Dresden, 2014.

[8] A. Sakowitz, M. Mihaescu, L. Fuchs, Turbulent flow mechanisms in mixing T-junctions by Large Eddy Simulations, International Journal of Heat and Fluid Flow 45 (2014) 135-146. doi:10.1016/j. ijheatfluidflow.2013.06.014.

[9] C. Walker, A. Manera, B. Niceno, M. Simiano, H.-M. Prasser, Steadystate RANS-simulations of the mixing in a T-junction, Nuclear Engineering and Design 240 (9) (2010) 2107 - 2115, experiments and CFD Code Applications to Nuclear Reactor Safety (XCFD4NRS). doi : 10.1016/j.nucengdes.2010.05.056. 
[10] B. Smith, J. Mahaffy, K. Angele, A CFD benchmarking exercise based on flow mixing in a T-junction, Nuclear Engineering and Design 264 (2013) 80 - 88, sI:NURETH-14. doi:10.1016/j.nucengdes.2013.02. 030 .

[11] Y. Yang, J. H. Walther, Y. Yan, C. Wen, CFD modeling of condensation process of water vapor in supersonic flows, Applied Thermal Engineering 115 (2017) 1357 - 1362. doi:10.1016/j.applthermaleng.2017.01. 047.

[12] C. Moses, G. Stein, On the growth of steam droplets formed in a laval nozzle using both static pressure and light scattering measurements, Journal of Fluids Engineering 100 (3) (1978) 311-322. doi:10.1115/1. 3448672 .

[13] L. Vyskocil, J. Schmid, J. Macek, CFD simulation of air-steam flow with condensation, Nuclear Engineering and Design 279 (2014) 147-157. doi:10.1016/j.nucengdes.2014.02.014.

[14] B. Semlitsch, M. Mihaescu, Flow phenomena leading to surge in a centrifugal compressor , Energy 103 (2016) 572 - 587. doi:10.1016/j. energy.2016.03.032.

[15] A. Broatch, J. Galindo, R. Navarro, J. García-Tíscar, A. Daglish, R. K. Sharma, Simulations and measurements of automotive turbocharger compressor whoosh noise, Engineering Applications of Computational Fluid Mechanics 9 (1). doi:10.1080/19942060.2015.1004788.

[16] CD-adapco, STAR-CCM+, release version 11.06.010 Edition (2016).

URL http: //www.cd-adapco.com

[17] D. Tarí, Effect of inlet configuration on the performance and durability of an automotive turbocharger compressor, Ph.D. thesis, Universitat Politècnica de València. Departamento de Máquinas y Motores Térmicos (June 2018). doi:10.4995/Thesis/10251/104410.

URL http://hdl.handle.net/10251/104410

[18] R. Navarro, Predicting Flow-Induced Acoustics at Near-Stall Conditions in an Automotive Turbocharger Compressor: A Numerical Approach, Springer, 2018. doi:10.1007/978-3-319-72248-1. 
[19] A. Broatch, J. Galindo, R. Navarro, J. García-Tíscar, Methodology for experimental validation of a CFD model for predicting noise generation in centrifugal compressors, International Journal of Heat and Fluid Flow 50 (2014) 134-144. doi:10.1016/j.ijheatfluidflow.2014.06.006.

[20] F. Mendonça, O. Baris, G. Capon, Simulation of Radial Compressor Aeroacoustics using CFD, in: Proceedings of ASME Turbo Expo 2012, no. GT2012-70028, ASME, 2012, pp. 1823-1832. doi:10.1115/ GT2012-70028.

[21] Y. Patel, T. Turunen-Saaresti, G. Patel, A. Grönman, Numerical investigation of turbulence modelling on condensing steam flows in turbine cascade, in: ASME Turbo Expo 2014: Turbine Technical Conference and Exposition, American Society of Mechanical Engineers, 2014, pp. V01BT27A035-V01BT27A035.

[22] J. Galindo, A. Tiseira, P. Fajardo, R. Navarro, Analysis of the influence of different real flow effects on computational fluid dynamics boundary conditions based on the method of characteristics, Mathematical and Computer Modelling 57 (7-8) (2013) 1957-1964, Public Key Services and Infrastructures EUROPKI-2010-Mathematical Modelling in Engineering \& Human Behaviour 2011. doi:10.1016/j.mcm.2012.01.016.

[23] B. Cabral, L. C. Leedom, Imaging vector fields using line integral convolution, in: Proceedings of the $20^{\text {th }}$ annual conference on Computer graphics and interactive techniques, ACM, 1993, pp. 263-270. doi:10.1145/166117.166151.

[24] Q. Zhang, M. Li, G. Li, S. Shao, P. Li, Transient emission characteristics of a heavy-duty natural gas engine at stoichiometric operation with EGR and TWC, Energy 132 (2017) 225 - 237. doi:10.1016/j .energy . 2017. 05.039 .

[25] X.-J. Yu, B.-J. Liu, Stereoscopic PIV measurement of unsteady flows in an axial compressor stage, Experimental Thermal and Fluid Science 31 (8) (2007) 1049 - 1060. doi:10.1016/j.expthermflusci.2006.11. 001 .

[26] S. K. Dahikar, M. J. Sathe, J. B. Joshi, Investigation of flow and temperature patterns in direct contact condensation using PIV, PLIF 
and CFD, Chemical Engineering Science 65 (16) (2010) 4606 - 4620. doi:10.1016/j.ces.2010.05.004.

[27] C. Espinoza, M. Simmons, F. Alberini, O. Mihailova, D. Rothman, A. Kowalski, Flow studies in an in-line Silverson 150/250 high shear mixer using PIV, Chemical Engineering Research and Designdoi:10. $1016 / j$.cherd.2018.01.028.

[28] A. Darisse, J. Lemay, A. Benaïssa, Investigation of passive scalar mixing in a turbulent free jet using simultaneous LDV and cold wire measurements, International Journal of Heat and Fluid Flow 44 (2013) 284 292. doi:10.1016/j.ijheatfluidflow.2013.06.012.

[29] A. Mychkovsky, D. Rangarajan, S. Ceccio, LDV measurements and analysis of gas and particulate phase velocity profiles in a vertical jet plume in a 2D bubbling fluidized bed: Part I: A two-phase LDV measurement technique, Powder Technology 220 (2012) 55 - 62, selected Papers from the 2010 NETL Multiphase Flow Workshop. doi: $10.1016 / \mathrm{j}$. powtec . 2011.09.027. 